\title{
Robust reconstruction of the discrete state for a class of nonlinear uncertain switched systems ${ }^{\sharp}$
}

\author{
N. Orani*, A. Pisano, M. Franceschelli, A. Giua, E. Usai \\ Department of Electrical and Electronic Engineering (DIEE), University of Cagliari, Pzza d'Armi, I-09123, Cagliari, Italy
}

\section{A R T I C L E I N F O}

\section{Article history:}

Received 30 November 2009

Accepted 27 October 2010

\section{Keywords:}

Nonlinear switched systems

Uncertain systems

Discrete mode reconstruction

Sliding mode observers

\begin{abstract}
A B S T R A C T
This paper presents an approach to the robust state reconstruction for a class of nonlinear switched systems affected by model uncertainties. Under the assumption that the continuous state is available for measurement, an approach is presented based on concepts and methodologies derived from the sliding mode control theory. With noisefree state measurements, the time needed for reconstructing the discrete state after a transition can be made arbitrarily small by sufficiently increasing a certain observer tuning parameter. Simulations and experiments, carried out on a three-tank laboratory setup, are presented and commented.
\end{abstract}

(C) 2010 Elsevier Ltd. All rights reserved.

\section{Introduction}

In the last decade the control community has devoted a great deal of attention to the study of switched systems. A switched system has a discrete dynamics represented by a finite state machine that evolves according to the occurrence of discrete events. To each discrete state (or "mode") a continuous dynamics is associated [1-6]. A problem of great interest is the reconstruction of the discrete state through the observation of measurable system outputs. The techniques developed in this framework can be applied to several problems where the discrete events are not observable. In the framework of discrete event systems, several approaches have been proposed to estimate the discrete state [7,8]. In a more general hybrid context, the discrete state estimation has been discussed in $[9,10]$.

The problem of invertibility of nonlinear systems [11], i.e. the problem of reconstructing the input and switching signal uniquely from given output and initial state, was addressed in [12] for the class of switched nonlinear systems affine in the control variables. In general, the problem of invertibility for switched systems, especially linear switched systems, has received considerable attention [13-16].

In [17] the equivalence between piecewise affine systems and a broad class of hybrid systems was proved and it was shown through counterexamples that observability and controllability properties cannot be easily deduced from those of the component linear subsystems.

In [18] the architecture of a hybrid observer consisting of both a discrete and a continuous state identification part is presented and it is shown that under certain assumptions the discrete state of the system is identified in a finite number of steps. In their approach the discrete observer is a DES system and continuous dynamics are linear and time invariant, it assumes that some events causing the dynamics switchings are observable.

\footnotetext{
The authors gratefully acknowledge the financial support from the FP7 European Research Projects "PRODI-Power plants Robustification by fault Diagnosis and Isolation techniques", grant no. 224233, and "DISC-Distributed Supervisory Control of Large Plants", grant no. 224498.

* Corresponding author. Tel.: +39070675 5760; fax: +39070 6755782.

E-mail addresses: n.orani@diee.unica.it (N. Orani), pisano@diee.unica.it (A. Pisano), mauro.franceschelli@diee.unica.it (M. Franceschelli), giua@diee.unica.it (A. Giua), eusai@diee.unica.it (E. Usai).
} 
In [19] the observability of piecewise linear systems is studied and some linear-algebraic characterization of some observability properties are given. Finally in [20] the observability of the continuous and discrete states of a class of linear hybrid systems is addressed and rank conditions on the structural parameters of the model are given.

In this paper we investigate the problem of discrete state reconstruction for switched systems building on the idea that a general class of switched systems can be modeled by nonlinear systems with an affine binary input representing the system discrete state.

Our work differs from [18] in that we assume that the switching signal is completely unobservable and only the continuous state of the system is accessible. Furthermore we address nonlinear, time-varying systems with model uncertainties.

Objective of the present work, based upon [21], is to reconstruct such a binary input despite bounded uncertainties affecting the system dynamics. To this end we propose a sliding mode based unknown input reconstruction technique, that exhibits remarkable properties of robustness against uncertainties and disturbances [22]. In [23] an overview of some recent results in the field of sliding mode based observers is presented.

In [24], within a distinct framework related to FDI, an approach to unknown input reconstruction was suggested based on a first-order sliding mode control technique. Such an approach could be used to reconstruct the discrete state of the switched system using the equivalent control principle through low pass filtering. However, it is well known that the methods based on low pass filtering can guarantee, at best, the asymptotic reconstruction of the discrete system state $[25,24,26]$.

Recently several methodologies have been proposed in the literature for the finite time reconstruction of unknown inputs based on higher-order sliding mode theory, via the so-called hierarchical HOSM observation approach [27,28]. In this paper a finite time observer based on the sub-optimal sliding mode algorithm will be suggested, which provides the exact unknown input reconstruction and a guaranteed convergence time in spite of model uncertainties.

The organization of the paper is as follows. Section 2 describes the considered class of uncertain switched systems. It is pointed out, in particular, that the considered class, that embeds both analog and binary terms, can capture switched dynamics of a certain degree of generality. Section 3 presents the proposed discrete state reconstruction scheme which is based on the second-order sliding mode (2-SM) approach. Section 4 introduces a case study of a physical example (a hydraulic three-tank system) that falls into the considered class of switched systems. Section 5 deals with the simulation results while in Section 6 we verify the effectiveness of the proposed scheme by real experiments using a laboratory-size three-tank system set up. Section 7 summarizes the attained results and draws possible lines for future improvements of the presented results.

\section{Problem formulation}

In this paper we examine the class of nonlinear systems that can be represented in the form:

$$
\dot{\mathbf{x}}(t)=\mathbf{G}(\mathbf{x}, \mathbf{u}, t)+\mathbf{D}(\mathbf{x}, \mathbf{u}, t) \delta(t)+\varepsilon(\mathbf{x}, t)
$$

where $\mathbf{x}(t) \in \mathbb{X} \subset \mathbb{R}^{n}$ is the continuous state, $\mathbf{u}(t) \in \mathbb{U} \subset \mathbb{R}^{p}$ is the continuous input, $\delta(t) \in\{0,1\}^{L}$ is a binary discrete input, $\mathbf{G}(\mathbf{x}, \mathbf{u}, t) \in \mathbb{R}^{n}$ and $\mathbf{D}(\mathbf{x}, \mathbf{u}, t) \in \mathbb{R}^{n \times L}$ are known vector fields, and $\varepsilon(\mathbf{x}, t) \in \mathbb{R}^{n}$ is an uncertain nonlinear term representing model mismatches and/or external disturbances.

According to a widely used terminology [29], the nonlinear system (1) can be classified as a system "affine" in the binary discrete input vector $\delta(t)$, and the problem tackled in this paper is the reconstruction of this vector.

\subsection{Assumptions}

We now specify the assumptions that the considered class of systems (1) must satisfy. In the following $\|\cdot\|$ denotes the 2-norm.

1. The continuous state $\mathbf{x}(t)$ is supposed to be fully measurable.

2. The time evolutions of the continuous state $\mathbf{x}$ and exogenous input $\mathbf{u}$ variables are confined in the compact domains $\mathbb{X}$ and $\mathbb{U}$.

3. The dimension $L$ of vector $\delta(t)$ must not exceed the dimension of the continuous state:

$$
L \leq n .
$$

4. The binary vector $\delta(t)$ is piecewise constant and there exists a minimal dwell time $t_{d}>0$ such that if $\left[t_{i}, t_{i+1}\right)$ is a maximal interval in which $\delta$ is constant it holds that $t_{i+1}-t_{i} \geq t_{d}$.

5. Matrices $\mathbf{G}(\mathbf{x}, \mathbf{u}, t), \mathbf{D}(\mathbf{x}, \mathbf{u}, t)$ are supposed to be known.

6. The full-rank matrix $\mathbf{D}(\mathbf{x}, \mathbf{u}, t)$ and the unmeasurable state dependent uncertainty or disturbance term $\varepsilon(\mathbf{x}, t)$ satisfy the next inequalities:

$$
\begin{aligned}
& \|\mathbf{D}(\mathbf{x}, \mathbf{u}, t)\| \leq D_{0} \quad\|\dot{\mathbf{D}}(\mathbf{x}, \mathbf{u}, t)\| \leq D_{1} \\
& \left\|\left[\mathbf{D}^{T}(\mathbf{x}, \mathbf{u}, t) \mathbf{D}(\mathbf{x}, \mathbf{u}, t)\right]^{-1} \mathbf{D}^{T}(\mathbf{x}, \mathbf{u}, t)\right\| \geq D_{2} \\
& \|\varepsilon(\mathbf{x}, t)\| \leq \bar{\varepsilon}_{0} \quad\|\dot{\varepsilon}(\mathbf{x}, t)\| \leq \bar{\varepsilon}_{1} .
\end{aligned}
$$

Note that no smoothness and norm-boundedness assumptions are made with respect to the vector field $\mathbf{G}(\mathbf{x}, \mathbf{u}, t)$. 
Assumption 1 could be considered quite restrictive since usually in observation problems only an output vector (having dimension lower than that of the state) is assumed to be available for measurements. It is worth noting, however, that the problem of estimating simultaneously the continuous and discrete state of a nonlinear and uncertain switched system is very challenging. In this work, we address the preliminary step of reconstructing the discrete state by assuming the measurement for the full continuous state vector, and the generalization to the output-feedback case, which is not trivial, calls for future investigations. Assumption 2 could be possibly relaxed by using different "global" versions of the suboptimal algorithm (see, e.g. [30]). Also, this extension is not trivial. Assumption 3 as well as Assumptions 4 and 5 is a structural requirement that cannot be removed. The latter, in particular, is necessary if one wants to reconstruct the binary vector exactly. Assumption 6 is mainly of technical nature, in the sense that an effective tuning of the proposed scheme can be carried out dispensing with the precise knowledge of the constants involved in (3)-(5). In summary, the overall set of Assumptions 1-6 appear to be mild enough to include systems of practical relevance, as confirmed by the successful experimental results.

\subsection{Comments on the considered class of systems}

We observe that the considered class of nonlinear systems (1) can also be seen as a switched system where the value of the binary discrete input determines a mode of evolution.

In particular the considered model is general enough to represent the following continuous-time switched nonlinear dynamics

$$
\dot{\mathbf{x}}(t)=\mathbf{G}_{\sigma(t)}(\mathbf{x}, \mathbf{u}, t)+\varepsilon(\mathbf{x}, t)
$$

where piecewise-constant integer function $\sigma(t) \in\{0, \ldots, k-1\}$ specifies the evolution mode (i.e., the discrete state) active at time $t$.

A simple systematic procedure to put system (6) into the form (1) is now given. Define the matrices $\mathbf{D}(\mathbf{x}, \mathbf{u}, t)$ and $\mathbf{G}(\mathbf{x}, \mathbf{u}, t)$ as follows (omitting the dependence of its entries from $x, u$ and $t$ )

$$
\begin{aligned}
& \mathbf{D}(\mathbf{x}, \mathbf{u}, t)=\left[\mathbf{G}_{1}-\mathbf{G}_{0} \mathbf{G}_{2}-\mathbf{G}_{0} \cdots \mathbf{G}_{k-1}-\mathbf{G}_{0}\right] \\
& \mathbf{G}(\mathbf{x}, \mathbf{u}, t)=\mathbf{G}_{0}(\mathbf{x}, \mathbf{u}, t) .
\end{aligned}
$$

Now let $L=k-1$ and define

$$
\delta(t)=\left[\delta_{1}(t), \ldots, \delta_{L}(t)\right]^{T}=\gamma(\sigma(t))=\left[\gamma_{1}(\sigma(t)), \ldots, \gamma_{L}(\sigma(t))\right]^{T}
$$

where $\gamma:\{0, k-1\} \rightarrow\{0,1\}^{L}$ is such that for $j=1,2, \ldots, L$

$$
\gamma_{j}(\sigma)= \begin{cases}1 & \text { if } \sigma=j \\ 0 & \text { otherwise. }\end{cases}
$$

One can readily verify that system (6) is equivalent to (1) and (7)-(9). At each instant of time $\sigma(t) \in\{0, \ldots, k-1\}$ defines a discrete state and function $\gamma$ maps the discrete state $\sigma(t)$ into an $L$-dimensional binary vector $\delta(t)$ which "encodes" the current discrete state. Note that more efficient encodings are also possible - in general with an $L$-dimensional binary vector it is possible to encode up to $2^{L}$ discrete states - provided that each discrete state $\sigma(t)$ is uniquely mapped into a binary vector $\delta(t)$, and vice versa.

According to Assumption 4 in the previous subsection, the only requirement on the evolution of the discrete state is that the time between two consecutive switchings must be greater than or equal to a fixed dwell time $t_{d}$.

\section{Discrete state observer design} form:

The proposed discrete state estimator (which assumes the knowledge of the continuous system state) takes the following

$$
\dot{\mathbf{z}}=\mathbf{G}(\mathbf{x}, \mathbf{u}, t)+\mathbf{w}(t)
$$

where $\mathbf{z}$ represents the observer state and $\mathbf{w}$ is an observer input to be designed. Let $\mathbf{e}=\mathbf{x}-\mathbf{z}$ be the observer error variable. Then, from (1) and (10), the observer error dynamics is

$$
\dot{\mathbf{e}}=\mathbf{D}(\mathbf{x}, \mathbf{u}, t) \delta(t)+\varepsilon(\mathbf{x}, t)-\mathbf{w}(t) .
$$

\subsection{Observer input design}

Our objective is to design an observer control vector $\mathbf{w}$ guaranteeing the finite time convergence to zero of $\mathbf{e}$ and $\dot{\mathbf{e}}$.

A second-order sliding mode based approach, that enables us to reconstruct the discrete state, will be proposed. It must be highlighted that, dissimilar to other works [31] within a distinct framework related to a fault diagnosis, such an approach, theoretically exact, exhibits a solution converging in finite time. 
Consider the second time derivative of the error variable $\mathbf{e}$

$$
\ddot{\mathbf{e}}=\dot{\mathbf{D}}(\mathbf{x}, \mathbf{u}, t) \delta(t)+\mathbf{D} \frac{\mathrm{d}}{\mathrm{d} t} \delta(t)-\dot{\varepsilon}(\mathbf{x}, t)-\dot{\mathbf{w}}(t)
$$

which can be rewritten in compact form as follows:

$$
\ddot{\mathbf{e}}=\varphi(\mathbf{x}, \mathbf{u}, t)-\dot{\mathbf{w}}(t) .
$$

The uncertain "drift term" $\boldsymbol{\varphi}(\cdot)=\left[\varphi_{1}(\cdot), \varphi_{2}(\cdot), \ldots, \varphi_{n}(\cdot)\right]^{T}$ takes the following form

$$
\boldsymbol{\varphi}(\mathbf{x}, \mathbf{u}, t)=\dot{\mathbf{D}}(\mathbf{x}, \mathbf{u}, t) \delta(t)+\mathbf{D} \frac{\mathrm{d}}{\mathrm{d} t} \delta(t)-\dot{\varepsilon}(\mathbf{x}, t)
$$

which depends on $\delta(t)$ that must be reconstructed.

Denote $\mathbf{v}(t)=\left[v_{1}, v_{2}, \ldots, v_{n}\right]^{T} \equiv-\dot{\mathbf{w}}(t), y_{i, 1}=e_{i}$ and $y_{i, 2}=\dot{e}_{i}$, where $e_{i}$ and $\dot{e}_{i}$ represent the $i$ th entry of vectors $\mathbf{e}$ and $\dot{\mathbf{e}}$. Then it is possible to rewrite system (13) in terms of $n$ decoupled single input subsystems having the following form

$$
\left\{\begin{array}{l}
\dot{y}_{i, 1}=y_{i, 2}, \quad i=1,2, \ldots, n \\
\dot{y}_{i, 2}=\varphi_{i}(\mathbf{x}, \mathbf{u}, t)+v_{i}
\end{array}\right.
$$

The problem is to find a set of control inputs $v_{i}$ stabilizing the uncertain SISO systems (15) in finite time. To solve this problem, the second-order sliding mode control approach $[32,33]$ appears to be particularly appropriate because systems (15) have relative degree two with respect to the inputs $v_{i}$ 's which are treated as auxiliary control variables. The control task is complicated by the two issues: (a) variables $y_{i, 2}(i=1,2, \ldots, n)$ are not measurable, and (b) the drift terms $\varphi_{i}(x, u, t)$ are uncertain.

Let the mode switching sequence of the hybrid dynamics have a dwell time $t_{d}$. This means that $t_{i+1}-t_{i} \geq t_{d}$, for $i \geq 0$. The main idea is to use the discontinuous controller in [34]. Under the condition that a constant upper bound $\Phi_{i}$ to the drift term magnitude can be computed

$$
\left|\varphi_{i}(x, u, t)\right| \leq \Phi_{i} \quad \forall t
$$

such a controller is able to stabilize the uncertain SISO systems (15) in a finite time $t^{*} \ll t_{d}$ starting from any initial conditions $\left(y_{i, 1}(0), y_{i, 2}(0)\right)$ norm bounded by arbitrarily large constants.

Denote as $t_{i}(i=1,2, \ldots)$ the switching instants at which the active dynamics is commuting. The discrete state $\sigma(t)$, and then also the vector $\delta(t)$, are piecewise constant during the time intervals $T_{i}=\left(t_{i}, t_{i+1}\right)$ between two adjacent mode switchings. The effect of the impulsive term $\frac{\mathrm{d}}{\mathrm{d} t} \delta(t)$ at the switching instants $t_{i}$ is a jump in the $(e, \dot{e})$ state trajectories of system (13), and in particular, from (12), it results $\|\dot{e}\|=\|D(x, u, t)\|\|\delta(t)\| \leq D_{0} \sqrt{n}$, since the Euclidean norm of the $n$-dimensional binary vector $\delta$ will never exceed the value $\sqrt{n}$. So, considering the single $i$ th decoupled subsystem (15), at the first switching instant $t_{1}$ the point $\left(y_{i, 1}\left(t_{1}\right), y_{i, 2}\left(t_{1}\right)\right)$ will leave the origin according to $\left(0, y_{i, 2}\left(t_{1}^{+}\right)\right)$with

$$
\left\|y_{i, 2}\left(t_{1}^{+}\right)\right\| \leq D_{0} .
$$

After a new transient whose duration can be made less than $t^{*}$ the system will be steered back to the origin. Thus, at any time $t \in\left[t_{1}+t^{*}, t_{2}\right)$ and any $i=1, \ldots, n$, the conditions $y_{i, 1}(t)=y_{i, 2}(t)=0$, i.e., $e(t)=\dot{e}(t)=0$ will be satisfied. The reasoning is repeated over all the successive switching intervals. The key point is the capability of the robust controller presented in [34], that will be specified in what follows, of steering to zero the SISO systems (15) arbitrarily fast during the time interval between two adjacent mode switchings.

Along any interval $T_{i} \equiv\left(t_{i-1}, t_{i}\right),(i=1,2, \ldots), t_{0} \equiv 0$, the drift term (14), considering (3)-(5), can be upper bounded by

$$
\|\varphi(x, u, t)\| \leq \bar{\Phi} \equiv D_{1} \sqrt{n}+\bar{\varepsilon}_{1}, \quad t \in\left(t_{i-1}, t_{i}\right) .
$$

The existence of such a constant upper bound allows for designing a robust controller featuring the desired finite time convergence properties. The next theorem outlines the main result by introducing the so-called "suboptimal" second-order sliding mode control algorithm [34,35], together with the tuning rules that allow us to obtain an arbitrarily fast convergence, which is a basic requirement of the present problem.

Theorem 1. Consider system (15) and the control law

$$
v_{i}(t)=-V_{M} \operatorname{sign}\left(y_{i, 1}(t)-\frac{1}{2} y_{i, 1}\left(\xi_{i, j}\right)\right) \quad \xi_{i, j} \leq t<\xi_{i, j+1} j=1,2, \ldots
$$

where $\xi_{i, 0}=t_{0} \equiv 0, \xi_{i, j}$ is the sequence of time instants at which $y_{i, 2}(t)=0$, and

$$
V_{M}=\Gamma \bar{\Phi} .
$$

Denote the sequence of switching instants as $t_{h}, h=1,2, \ldots$. Then there is $\Gamma^{*}$ such that, for any $\Gamma \geq \Gamma^{*}$, the following conditions are provided.

$$
y_{i, 1}(t)=y_{i, 2}(t)=0, \quad t \in\left(t_{h}+t^{*}, t_{h+1}\right)
$$

where $i=1,2, \ldots, n$ and with $t^{*}$ being arbitrarily small. 
Proof of Theorem 1. The proof exploits the basic convergence properties of the suboptimal second-order sliding mode control algorithm [35]. Let us consider the single $i$ th decoupled subsystem (15) and denote with $t_{1}$ the first switching instant. At $t=t_{1}$, when the $y_{i, 1}$ and $y_{i, 2}$ variables are both assumed zero, on the basis of (17), we can infer that $y_{i, 2}$ undergoes a jump that, for the worst case, leads the system states $\left(y_{i, 1}\left(t_{1}^{+}\right), y_{i, 2}\left(t_{1}^{+}\right)\right)$in the point $\left(0, D_{0}\right)$. Starting from here, after a time $t_{M 1} \leq \frac{D_{0}}{\bar{\Phi}(\Gamma-1)}$, the extremal point $\left(\frac{D_{0}^{2}}{2 \bar{\Phi}(\Gamma-1)}, 0\right)$ is reached. The suboptimal control strategy, with the additional constraint $\Gamma>2$, causes the generation of a sequence of states, with coordinates $\left(y_{i, 1_{M 1}}, 0\right)$, featuring the following contraction property:

$$
\left|y_{i, 1_{M 1+j}}\right| \leq \alpha\left|y_{i, 1_{M 1}}\right|, \quad j=1,2, \ldots, \alpha=\frac{1}{\Gamma-1} \in[0,1) .
$$

Such a sequence converges to the origin at finite time $t^{*}$ that can be made arbitrarily small opportunely tuning the gain parameter $\Gamma$. By imposing the following condition $t^{*}<t_{d}$,

$$
\frac{D_{0}}{\bar{\Phi}(\Gamma-1)}+\frac{2 \Gamma \bar{\Phi} \sqrt{\frac{D_{0}^{2}}{2 \bar{\Phi}(\Gamma-1)}}}{(\Gamma-1) \sqrt{\bar{\Phi}(\Gamma+1)}} \frac{1}{1-\sqrt{\frac{1}{\Gamma-1}}}<t_{d} .
$$

From (23) it follows the rule for selecting $\Gamma$ in such a way that the convergence time $t^{*}$ fulfills the inequality $t^{*}<t_{d}$. An admissible interval for $\Gamma$ exists due to the fact that the left hand side, i.e. $t^{*}$ converges to zero for $\Gamma \longrightarrow \infty$. On the basis of previous observations, denoting by $\Gamma^{*}$ the value of $\Gamma$ such that $t^{*}=t_{d}$, choosing the tuning parameter $\left.\left.\Gamma \in\right] \Gamma^{*}, \infty\right)$, the condition $t^{*}<t_{d}$ is guaranteed.

\subsection{Discrete state reconstruction}

It was shown in the previous section that there is $t^{*}$ such that, in every "inter-switching" interval $T_{i} \equiv\left(t_{i-1}, t_{i}\right)$ the next conditions hold

$$
\mathbf{e}=\dot{\mathbf{e}}=0, \quad t \in\left[t_{i-1}+t^{*}, t_{i}\right) .
$$

From definition (11) of $\dot{\mathbf{e}}$, its zeroing implies that

$$
\mathbf{D}(\mathbf{x}, \mathbf{u}, t) \delta(t)+\varepsilon(\mathbf{x}, t)-\mathbf{w}(t)=0 .
$$

Notice that the observer input $\mathbf{w}(t)$ is obtained by integrating the discontinuous signal $\mathbf{v}(t)$, whose sign switches at very high (theoretically infinite) frequency (Zeno behaviour), then $\mathbf{w}(t)$ is a continuous signal.

By neglecting the uncertainty $\varepsilon(\mathbf{x}, t)$ in (25) it yields naturally the following reconstruction formula that defines a "non-thresholded" estimate of the binary vector $\delta$.

$$
\tilde{\delta}(t)=\left[\mathbf{D}^{T}(\mathbf{x}, \mathbf{u}, t) \mathbf{D}(\mathbf{x}, \mathbf{u}, t)\right]^{-1} \mathbf{D}^{T}(\mathbf{x}, \mathbf{u}, t) \mathbf{w}(t) .
$$

The non-thresholded estimate is not robust against the uncertainty $\varepsilon(\mathbf{x}, t)$. By (25), the estimation error $\tilde{\delta}(t)-\delta(t)$ will be such that

$$
\|\tilde{\delta}(t)-\delta(t)\| \leq\left\|\left[\mathbf{D}^{T}(\mathbf{x}, \mathbf{u}, t) \mathbf{D}(\mathbf{x}, \mathbf{u}, t)\right]^{-1} \mathbf{D}^{T}(\mathbf{x}, \mathbf{u}, t)\right\|\|\varepsilon\| .
$$

It can fruitfully exploit the binary nature of the vector $\delta(t)$ by introducing a thresholding that rounds the value of $\tilde{\delta}(t)$ to the closest integer value between 0 and 1 . It yields the "thresholded" estimate $\hat{\delta}(t)$ defined according to

$$
\hat{\delta}_{i}(t)= \begin{cases}1 & \tilde{\delta}_{i}(t)>0.5 \\ 0 & \tilde{\delta}_{i}(t) \leq 0.5\end{cases}
$$

where $\tilde{\delta}_{i}(t)$ and $\hat{\delta}_{i}(t)$ are the $i$ th entries of vectors $\tilde{\delta}(t)$ and $\hat{\delta}(t)$, respectively. The thresholded estimate results to be robust against any error $\left(\tilde{\delta}_{i}(t)-\delta_{i}(t)\right)$ less, in magnitude, than 0.5 . Thus it can be explicitly given a bound to the maximal tolerated magnitude for the uncertainty term.

From the requirement that $\|\tilde{\delta}(t)-\delta(t)\| \leq 0.5$ it yields by (27), (4) and (5) the following maximal acceptable bound for the norm of the uncertainty term

$$
\|\varepsilon(\mathbf{x}, t)\| \leq \bar{\varepsilon}_{0} \leq \frac{0.5}{D_{2}} .
$$

The fulfillment of (29) guarantees the insensitivity of the estimate $\hat{\delta}$ against the uncertainty, namely the condition

$$
\hat{\delta}(t)=\delta(t), \quad t \in\left[t_{i-1}+t^{*}, t_{i}\right), i=1,2, \ldots
$$

Lemma 1. Under the condition that the norm of the uncertain term $\varepsilon(\mathbf{x}, t)$ fulfills the restriction (29), the proposed estimation procedure given by (26), (28) provides the exact reconstruction of the binary vector $\delta$ in the time intervals $t \in\left[t_{i-1}+t^{*}, t_{i}\right)$, according to (30). 


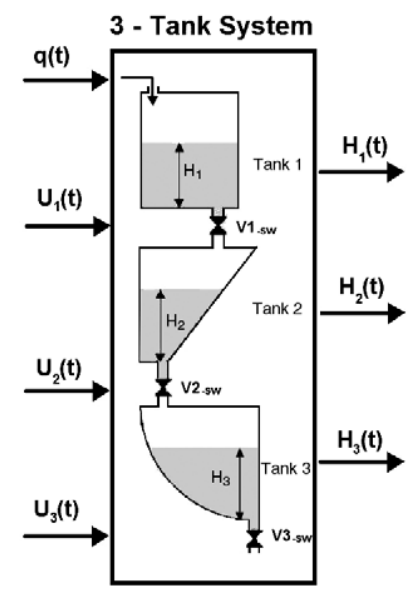

Fig. 1. System inputs and outputs.

Remark 1. The requirement of providing the observer convergence within the arbitrarily small transient time $t^{*} \ll t_{d}$ would correspond, in the linear context, to locating the eigenvalues of the error dynamics far away from the origin. Generally, this strongly deteriorates the robustness against the measurement noise of the resulting linear "high gain" observer. It can be argued, due to the analysis made in [36,32], that the magnification of the noise in the considered 2-SMC observer could be less severe than in the linear observer counterpart. This topic will be addressed in more detail in next research activities.

\section{The three-tank system case study}

The three-tank water process is regarded as a valuable setup for investigating nonlinear multivariable control as well as fault diagnosis schemes [37]. Let us show that it can be modeled as a switched affine system according to the general formulation (1).

The vertical multi-tank system that we shall consider is composed of three tanks of different shapes interconnected as shown in Fig. 1, a water inflow $q(t)$ that supplies the upper tank no. 1 and three on-off valves $V 1_{s w}, V 2_{s w}, V 3_{s w}$ that determine whether an outflow from each Tank exists or not. The on-off state of the three valves define the 8 possible operating modes of the considered system.

Refer to the schematic representation in Fig. 1. Signal $q(t)$, the inflow to the upper tank, represents a measurable input to the system, the binary signals $U_{1}, U_{2}$ and $U_{3}$ are the unknown states of the on-off valves, and $H_{1}, H_{2}$ and $H_{3}$ are the water levels which represent the continuous state of the three-tank system. It is the objective of the present work to present a scheme for reconstructing the states of the three on-off valves by assuming the knowledge of the water levels and of the input inflow $q(t)$ to the upper tank.

Let us model the three-tank system. The flow balance equations lead to

$$
\begin{aligned}
& \dot{V}_{1}=q(t)-C_{1}(t) \sqrt{H_{1}} \\
& \dot{V}_{2}=C_{1}(t) \sqrt{H_{1}}-C_{2}(t) \sqrt{H_{2}} \\
& \dot{V}_{3}=C_{2}(t) \sqrt{H_{2}}-C_{3}(t) \sqrt{H_{3}}
\end{aligned}
$$

where $V_{1}, V_{2}, V_{3}$ correspond to the actual volume of water in the three tanks and $C_{1}(t), C_{2}(t)$ and $C_{3}(t)$ are the outflow nonnegative coefficients of the respective valves that can be adjusted. Obviously when the generic $i$ th valve is in the fully closed position, the corresponding coefficient $C_{i}$ is equal to zero, vice versa if the valve is in fully opened condition $C_{i}=C_{i}^{*}$. Therefore it seems appropriate to represent such outflow coefficients by the notation $C_{i}(t)=C_{i}^{*} U_{i}(t)$, where $U_{i}(t) \in[0,1]$ is time varying and represents the relative actual valve opening.

It results

$$
C_{i}(t)= \begin{cases}0 & \text { when valve } V i_{s w} \text { is OFF } \\ C_{i}^{*} & \text { when valve } V i_{s w} \text { is ON. }\end{cases}
$$

Further, the following simple relationships hold

$$
\dot{V}_{i}=\beta_{i}\left(H_{i}\right) \dot{H}_{i}, \quad i=1,2,3
$$

where $\beta_{i}\left(H_{i}\right)(i=1,2,3)$ represents the cross-sectional area of the $i$ th tank $i$ at the level height $H_{i}$. It yields the simple model

$$
\dot{H}_{1}=\frac{1}{\beta_{1}\left(H_{1}\right)}\left[q(t)-C_{1}^{*} \sqrt{H_{1}} U_{1}(t)\right]
$$




$$
\begin{aligned}
& \dot{H}_{2}=\frac{1}{\beta_{2}\left(H_{2}\right)}\left[C_{1}^{*} \sqrt{H_{1}} U_{1}(t)-C_{2}^{*} \sqrt{H_{2}} U_{2}(t)\right] \\
& \dot{H}_{3}=\frac{1}{\beta_{3}\left(H_{3}\right)}\left[C_{2}^{*} \sqrt{H_{2}} U_{2}(t)-C_{3}^{*} \sqrt{H_{3}} U_{3}(t)\right] .
\end{aligned}
$$

Collecting into a binary vector the discrete states of the on-off valves as follows

$$
\delta(t)=\left[U_{1}(t), U_{2}(t), U_{3}(t)\right]^{T} \in\{0,1\}^{3}
$$

it is straightforward to rewrite the model (36)-(38) in the form (1) with $\mathbf{x}=\left[H_{1}, H_{2}, H_{3}\right]^{T}, \mathbf{u}=q(t)$ and

$$
\begin{aligned}
\mathbf{G}(\mathbf{x}, \mathbf{u}, t) & =\left[\begin{array}{c}
q(t) \\
\beta_{1}\left(H_{1}\right) \\
0 \\
0
\end{array}\right] \\
\mathbf{D}(\mathbf{x}, \mathbf{u}, t) & =\left[\begin{array}{ccc}
-\frac{C_{1} * \sqrt{H_{1}}}{\beta_{1}\left(H_{1}\right)} & 0 & 0 \\
\frac{C_{1}^{*} \sqrt{H_{1}}}{\beta_{2}\left(H_{2}\right)} & -\frac{C_{2}{ }^{*} \sqrt{H_{2}}}{\beta_{2}\left(H_{2}\right)} & 0 \\
0 & \frac{C_{2}^{*} \sqrt{H_{2}}}{\beta_{3}\left(H_{3}\right)} & -\frac{C_{3}^{*} \sqrt{H_{3}}}{\beta_{3}\left(H_{3}\right)}
\end{array}\right] .
\end{aligned}
$$

According to the notation introduced in Section 2, it must be highlighted that in our case a particular instance for (39) represents one of the possible $k=8$ discrete states $\sigma(t)$ associated to a respective nonlinear dynamic in which the threetank system could be found. A possible realization for $\sigma(t)$ could be as follows

$$
\sigma(t)=\delta_{1}(t) \cdot 2^{2}+\delta_{2}(t) \cdot 2^{1}+\delta_{3}(t) \cdot 2^{0} .
$$

In the derived three-tank system the dimension $L$ of vector $\delta(t)$ is $L=3$ which does not exceed the dimension $n=3$ of the continuous state, as required in assumption (2).

The assumptions (3) on the matrix $\mathbf{D}(\mathbf{x}, \mathbf{u}, t)$ are trivially satisfied if the water levels $H_{1}(t), H_{2}(t), H_{3}(t)$ remain strictly positive during the observation process. Further assumption (4) requires that the square matrix $\mathbf{D}(\mathbf{x}, \mathbf{u}, t)$ be nonsingular. Since

$$
\operatorname{det} \mathbf{D}(\mathbf{x}, \mathbf{u}, t)=-\frac{C_{1}{ }^{*} C_{2}{ }^{*} C_{3}{ }^{*}}{\beta_{1}\left(H_{1}\right) \beta_{2}\left(H_{2}\right) \beta_{3}\left(H_{3}\right)} \sqrt{H_{1} H_{2} H_{3}}
$$

again assumption (4) is fulfilled if none of the water levels become zero during the observation process. Assuming that an appropriate closed-loop supervisory system has been designed, capable of guaranteeing that $H_{i}(t) \geq H_{i}^{*}>0, i=1,2,3$, the proposed observer can provide for the reconstruction of the binary signal vector $\delta(t)$.

An additive error term $\varepsilon(\mathbf{x}, t)$ may take into account possible discrepancies between the actual and nominal system models as well as possible external disturbances. It is stated in Lemma 1 that the discrete state can still be reconstructed exactly provided that the norm of $\varepsilon(\mathbf{x}, t)$ is sufficiently small.

It is worth noting that the discrete state $\sigma(t) \in\{0,1, \ldots, 7\}$ can be reconstructed from the thresholded estimates $\hat{\delta}_{1}(t), \hat{\delta}_{2}(t), \hat{\delta}_{3}(t)$, according to (42), by means of the following expression

$$
\hat{\sigma}(t)=\hat{\delta}_{1}(t) \cdot 2^{2}+\hat{\delta}_{2}(t) \cdot 2^{1}+\hat{\delta}_{3}(t) \cdot 2^{0} .
$$

\section{Simulation results}

The effectiveness of the suggested discrete state observer is now studied by means of some simulative analysis conducted on the three-tank model (36)-(38). The inflow input $q(t)$ and the binary state $\delta(t)$ have been selected in such a way that the tanks never become empty, that would cause the loss of observability for the system.

The cross-sectional area functions $\beta_{1}\left(H_{1}\right), \beta_{2}\left(H_{2}\right), \beta_{3}\left(H_{3}\right)$ have the following analytical expressions which depend on the particular, different, shapes for the considered three-tank system represented in Fig. 1:

$$
\begin{aligned}
& \beta_{1}\left(H_{1}\right)=a w \\
& \beta_{2}\left(H_{2}\right)=c w+b w H_{2} \\
& \beta_{3}\left(H_{3}\right)=w \sqrt{R^{2}-\left(R-H_{3}\right)^{2}}
\end{aligned}
$$

where $a, b, c, w, R$ are appropriate constant geometric parameters.

The parameter values used in the simulations, evaluated by means of an identification procedure, are reported in Table 1. 
Table 1

\begin{tabular}{lll}
\multicolumn{2}{l}{ Identified parameters. } \\
\hline Parameter & Value & Unit \\
\hline$C_{1}{ }^{*}$ & $6.1 * 10^{-5}$ & \\
$C_{2}{ }^{*}$ & $6.5 * 10^{-5}$ & \\
$C_{3}{ }^{*}$ & $6.4 * 10^{-5}$ & \\
$a$ & 0.035 & $\mathrm{~m}$ \\
$w$ & 0.035 & $\mathrm{~m}$ \\
$b$ & 0.348 & $\mathrm{~m}$ \\
$c$ & 0.1 & $\mathrm{~m}$ \\
$R$ & 0.365 & $\mathrm{~m}$ \\
\hline
\end{tabular}
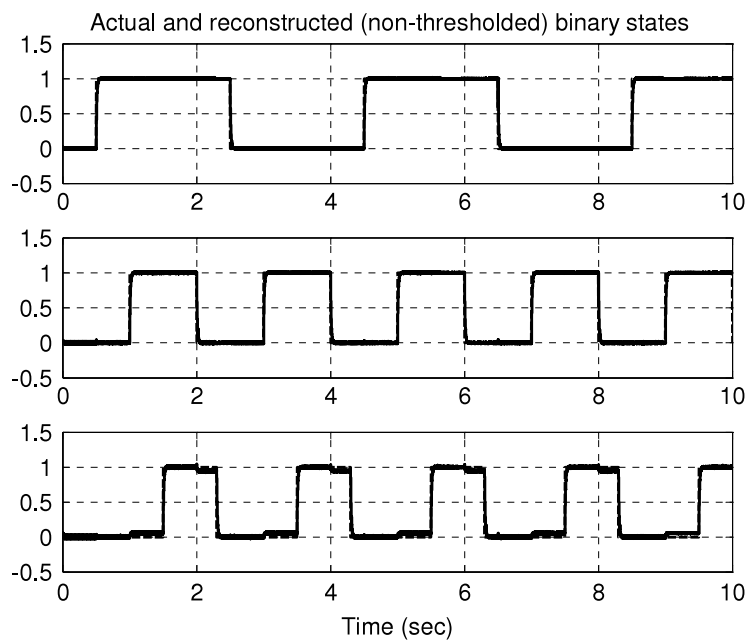

Fig. 2. TEST 1. $\delta_{i}(t)$ vs. the corresponding non-thresholded estimations $\tilde{\delta}_{i}(t)$. From top to bottom: $i=1,2,3$.

Euler integration method with the fixed sampling time $T_{s}=0.001 \mathrm{~s}$ has been used. A disturbance vector with elements of the form

$$
\varepsilon_{i}(\mathbf{x}, t)=0.1\left(\left|H_{1}(t)\right|+\left|H_{2}(t)\right|+\left|H_{3}(t)\right|\right) \sin (\omega t), \quad i=1,2,3
$$

is considered, and a band-limited additive white noise is added to the level measurements $H_{1}, H_{2}, H_{3}$. The binary signal inputs $U_{1}(t), U_{2}(t), U_{3}(t)$ defining the discrete state of the system have been selected as shown in the plot of the next Fig. 2 (the same profile for all the simulation tests has been used). It can be noted that a dwell time of $0.5 \mathrm{~s}$ has been used.

In TEST 1, the disturbance vector $\varepsilon(\mathbf{x}, t)$ and the measurement noise are not included. The plots in Fig. 2 show the actual $\delta_{i}(t)$ values together with the non-thresholded reconstructed ones $\tilde{\delta}_{i}(t)$. Fig. 3 makes the same comparison by considering the thresholded reconstructed values $\hat{\delta}_{i}(t)$. Fig. 4 shows the actual and reconstructed discrete states $\sigma(t)$ and $\hat{\sigma}(t)$. It can be seen that the suggested method provides a prompt identification of the active mode.

In TEST 2 it is shown that by increasing the $V_{M}$ observer parameter an arbitrarily fast identification of the current mode after the mode switchings can be achieved. To this end three different values of $V_{M}$ have been considered, and a zoom across some switching instant is made in Fig. 5 . The differences in the transient duration confirm the expected performance.

In TEST 3, disturbances and measurement noise are considered. Fig. 6 shows that signals $\delta_{i}(t)$ are corrupted by the noise as compared with the TEST 1 . But, since the resulting errors are less than 0.5 , the successive thresholding removes the errors and recovers the correct discrete state estimates according to Lemma 1 (see Fig. 7).

\section{Experimental results}

Experimental results using the three-tank laboratory-size apparatus by Inteco [38] are presented and commented in this section. A picture of the experimental setup is shown in Fig. 8.

The multi-tank system is interfaced with an external PC-based data acquisition and control system. The development of, both, the controller and observer systems is made in the MATLAB/Simulink environment, and the associated executable code is automatically generated by the RTW rapid prototyping environment. The water levels are measured by means of piezo-resistive pressure transducers and acquired by a multipurpose DAC I/O board. 

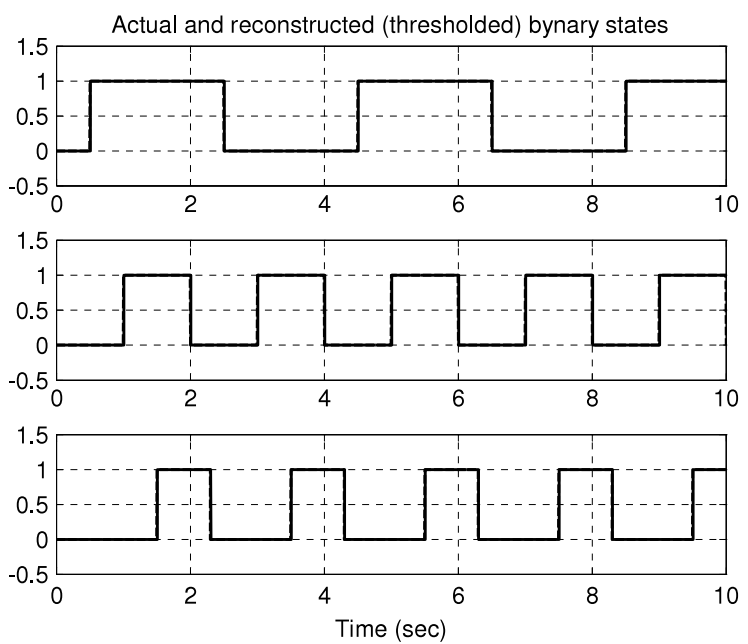

Fig. 3. TEST 1. $\delta_{i}(t)$ vs. the corresponding thresholded estimations $\hat{\delta}_{i}(t)$. From top to bottom: $i=1,2,3$.

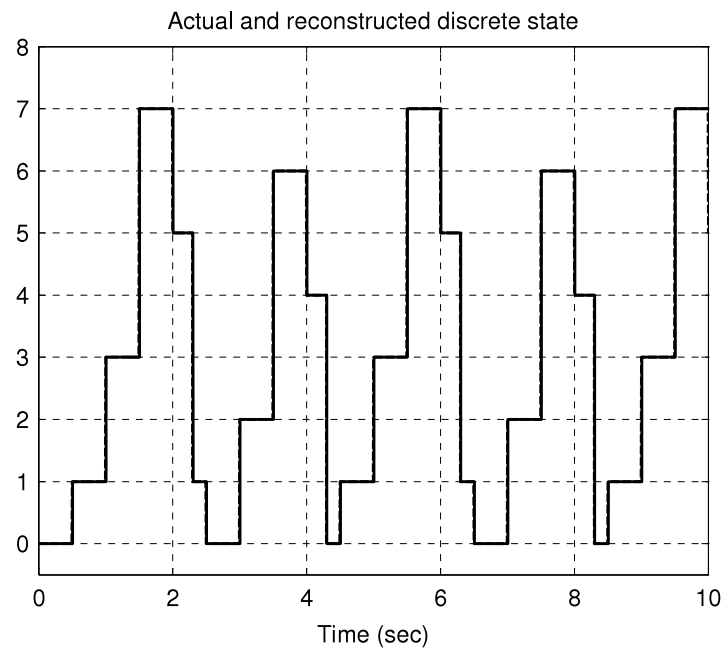

Fig. 4. TEST 1. Actual $\sigma(t)$ and reconstructed $\hat{\sigma}(t)$.

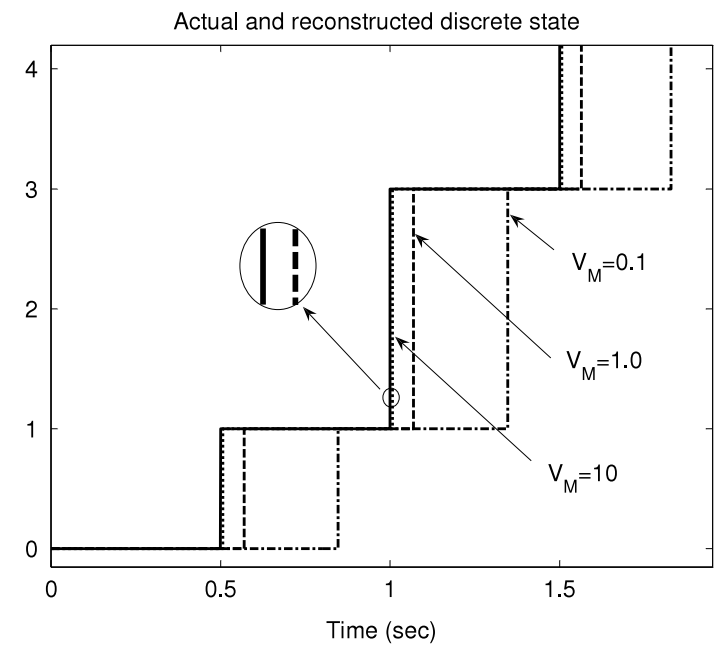

Fig. 5. TEST 2. $\sigma(t)$ (solid line) and $\hat{\sigma}(t)$ (dashed lines) using different values for the $V_{M}$ observer gain. 

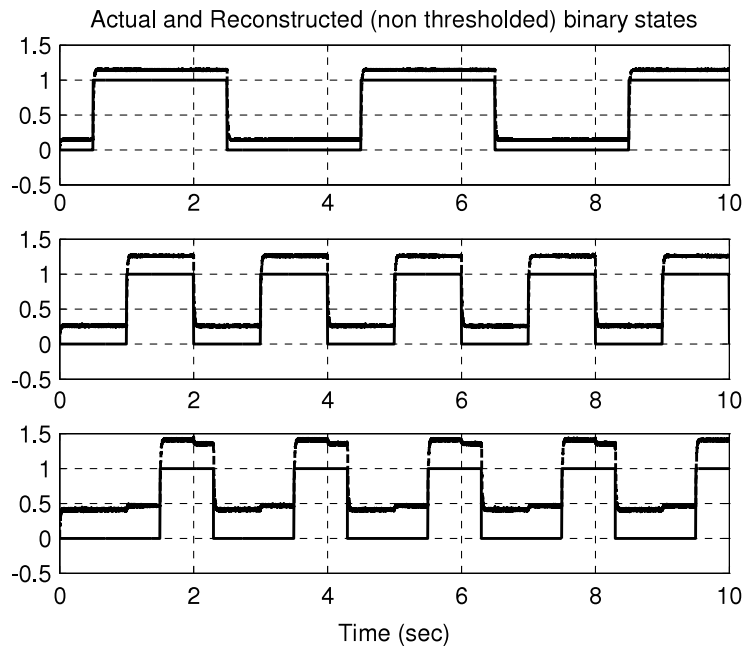

Fig. 6. TEST 3. Actual $\delta_{i}(t)$ and non-thresholded reconstructed $\tilde{\delta}_{i}(t)$ discrete inputs. From top to bottom: $i=1,2,3$.

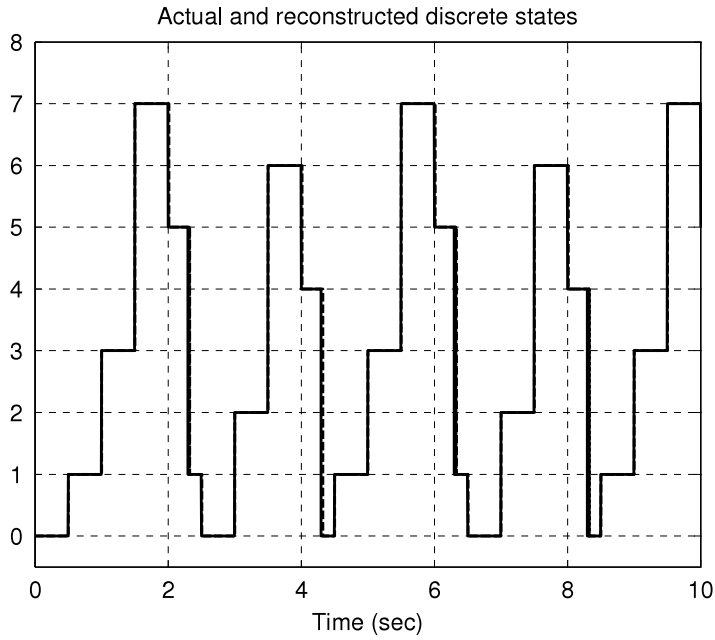

Fig. 7. TEST 3. Actual $\sigma(t)$ and reconstructed $\hat{\sigma}(t)$ discrete states.

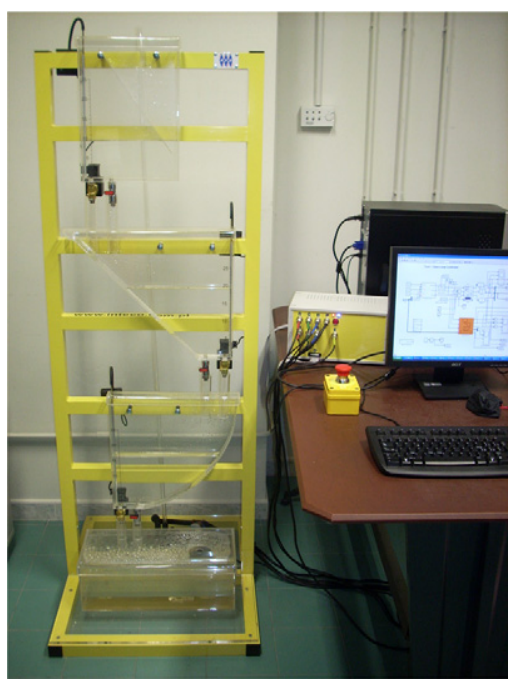

Fig. 8. The laboratory-size three-tank system. 


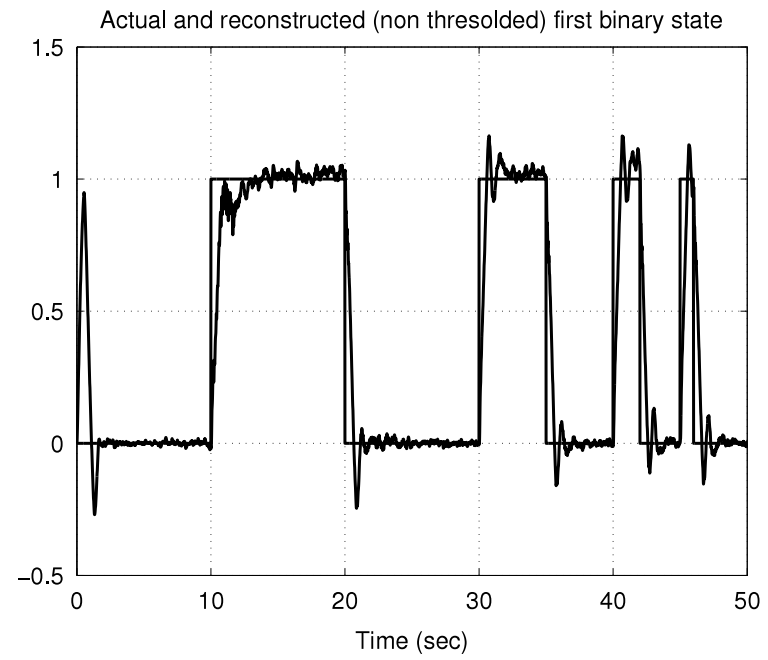

Fig. 9. Actual $\delta_{1}(t)$ and non-thresholded reconstructed $\tilde{\delta}_{1}(t)$ discrete inputs.

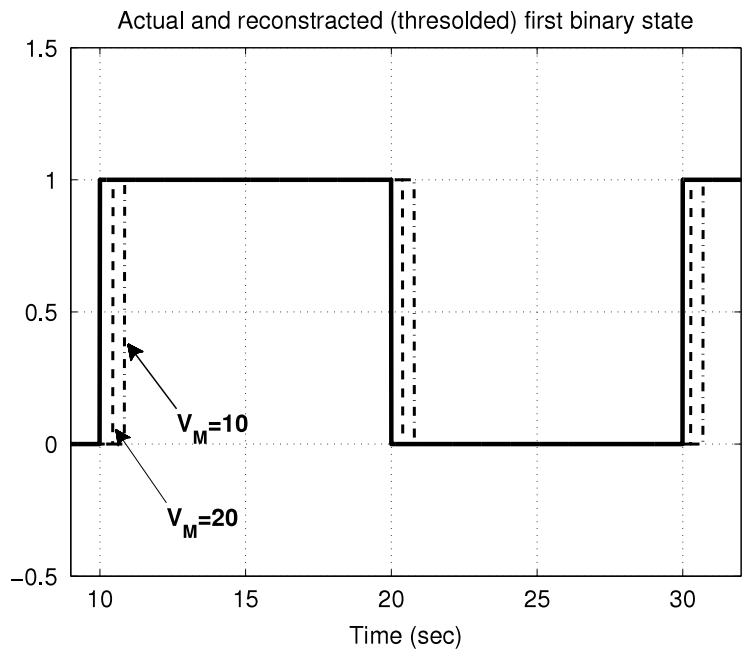

Fig. 10. $\delta_{1}(t)$ (solid line) and $\hat{\delta}_{1}(t)$ (dashed lines) using different values for the $V_{M}$ observer gain.

There are four control signals generated by the DAC board: the three binary control signals $U_{1}(t), U_{2}(t), U_{3}(t)$ for the on-off valves $V 1_{s w}, V 2_{s w}, V 3_{s w}$ and the pump control signal $q(t)$. As previously described, the on-off states of the three valves define 8 possible operating modes for the considered system.

The identification of the plant parameters has been carried out in order to minimize the discrepancies between the real process and its mathematical model (36)-(38).

The dimensional parameters $a, w, b, c, R$ have been directly measured, and suitable identification procedures, based on independent tests on each tank, have been implemented. The obtained values are reported in Table 1.

The closed-loop control system is based on anti-wind-up PI controllers. The sampling time is $0.01 \mathrm{~s}$. In Fig. 9 a generic trend of the binary state $\delta_{1}(t)$ of valve $V 1_{s w}$ and the corresponding reconstructed one $\tilde{\delta}_{1}(t)$ are shown. It must be highlighted that, after an initial transient time, the reconstructed signal matches quite precisely the signal $\delta_{1}(t)$.

Fig. 10 shows how, after the thresholding procedure (28), apart from a transient time depending on the magnitude $V_{M}$ of the corresponding injection signal, the current mode can be precisely reconstructed. In particular, as shown in Fig. 10, two different values for $V_{M}$ have been used, and as expected, by increasing $V_{M}$ the transient time can be reduced.

It must be highlighted that differently from what stated in the theoretical analysis, the transient time cannot be made arbitrarily small. This is due to several reasons, like discretization and noise effect. Furthermore, sensors and actuators are unavoidably affected by unmodeled dynamic effects.

The plots in Fig. 11 show the actual $\delta_{i}(t)$ values together with the thresholded reconstructed ones $\hat{\delta}_{i}(t)$.

Fig. 12 shows the actual and reconstructed discrete states $\sigma(t)$ and $\hat{\sigma}(t)$. It can be seen that the suggested method provides a prompt identification of the active mode. 

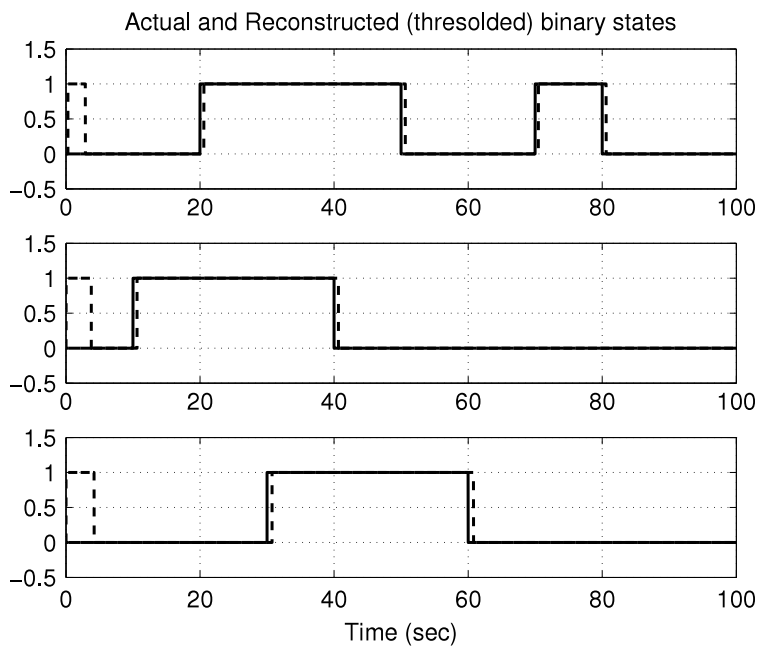

Fig. 11. $\delta_{i}(t)$ vs. the corresponding thresholded estimations $\hat{\delta}_{i}(t)$. From top to bottom: $i=1,2,3$.

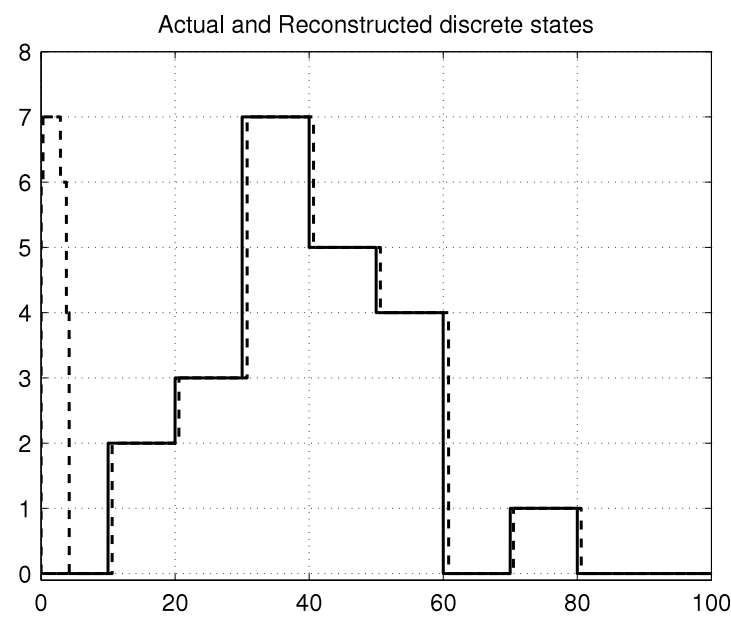

Fig. 12. TEST 3. Actual $\sigma(t)$ and reconstructed $\hat{\sigma}(t)$ discrete state.

\section{Conclusions}

A scheme for the reconstruction of the discrete state in a class of nonlinear uncertain switched systems has been proposed. Key ingredients of the proposed approach are the use of a second-order sliding mode observer approach in the presence of an impulsive drift term, that as far as we know was never studied before, followed by a thresholding procedure that allows to exactly reject the effect of a sufficiently small uncertainty term. Robustness against significant classes of disturbances is guaranteed by the proposed procedure that has been verified by simulations and experiments. Next, activities could be devoted to relaxing the requirement of knowing the continuous state by providing the simultaneous reconstruction of continuous and discrete states by output measurements, and to enlarge the observed system class by including, for instance, switching dynamics which are not affine on the binary vector $\delta(t)$.

\section{References}

[1] R. Alur, C. Courcoubetis, T.A. Henzinger, Pei-Hsin Ho, Hybrid automata: An algorithmic approach to the specification and verification of hybrid systems, Lecture Notes in Computer Science 736 (2003) 209-229.

[2] M.S. Branicky, V.S. Borkar, S.K. Mitter, A unified framework for hybrid control: model and optimal control theory, IEEE Transactions on Automatic Control 43 (1) (1998).

[3] D. Liberzon, A.S. Morse, Basic problems in stability and design of switched systems, Control Systems Magazine, IEEE 19 (5) (1999) 59-70.

[4] J. Arjan, van der Schaft, Hans Schumacher, An introduction to hybrid dynamical systems, Lecture Notes in Control and Information Sciences 251 (2000).

[5] R. Goebel, R.G. Sanfelice, A.R. Teel, Hybrid dynamical systems, IEEE Control Systems Magazine 29 (2) (2009) 28-93.

[6] H. Lin, P.J. Antsaklis, Stability and stabilizability of switched linear systems: a survey of recent results, IEEE Transactions on Automatic Control 54 (2) (2009) 308-322.

[7] A. Giua, C. Seatzu, Observability of place/transition nets, IEEE Transactions on Automatic Control 47 (9) (2002) 1424-1437.

[8] D. Corona, A. Giua, C. Seatzu, Marking estimation of Petri nets with silent transitions, IEEE Transactions on Automatic Control 52 (9) (2007) $1695-1699$. 
[9] W. Wan, L. Li, D. Zhou, K. Liu, Robust state estimation and fault diagnosis for uncertain hybrid nonlinear systems, Nonlinear Analysis: Hybrid Systems 1 (2007).

[10] M.D. Di Benedetto, S. Di Gennaro, A. D'Innocenzo, Discrete state observability of hybrid systems, International Journal of Robust and Nonlinear Control 19 (14) (2009) 1564-1580, Special Issue on "Observability and Observer-Based Control of Hybrid Systems", 2009.

[11] R. Hirschorn, Invertibility of multivariable nonlinear control systems, IEEE Transactions on Automatic Control 24 (6) (1979) 855-865.

[12] A. Tanwani, D. Liberzon, Invertibility of nonlinear switched systems, in: 47th IEEE Conference on Decision and Control, Cancun, Mexico, Dec. 2008.

[13] S. Sundaram, C.N. Hadjicostis, Designing stable inverters and state observers for switched linear systems with unknown inputs, in: 45th IEEE Conference on Decision and Control, San Diego, CA, USA, Dec. 2006.

[14] L. Vu, D. Liberzon, Invertibility of switched linear systems, Automatica 44 (4) (2008) 949-958.

[15] D. Liberzon, Switching in Systems and Control, Birkhauser, 2003.

[16] A. Alessandri, P. Coletta, Design of luenberger observers for a class of hybrid linear systems, in: HSCC'01: Proceedings of the 4th International Workshop on Hybrid Systems, 2001.

[17] A. Bemporad, G. Ferrari-Trecate, R. Morari, Observability and controllability of piecewise affine and hybrid systems, IEEE Transactions on Automatic Control 45 (10) (2000) 1864-1876.

[18] A. Balluchi, L. Benvenuti, A.L. Sangiovanni-Vincentelli, Observers for hybrid systems with continuous state resets, in: Proc. of the 10th Mediterranean Conference on Control and Automation, Lisbon, Portugal, July 2002.

[19] M. Babaali, M. Egerstedt, On the observability of piecewise linear systems, in: Proc. of IEEE Conference on Decision and Control, vol. 12004, pp. 26-31.

[20] R. Vidal, A. Chiuso, S. Soatto, Observability and identifiability of jump linear systems, in: Proc. of IEEE Conference on Decision and Control, vol. 4, 2002, pp. 3614-3619.

[21] N. Orani, A. Pisano, M. Franceschelli, A. Giua, E. Usai, Robust reconstruction of the discrete state for a class of nonlinear uncertain switched systems, in: ADHS09: 3rd IFAC Conference on Analysis and Design of Hybrid Systems. Zaragoza, Spain, September 2009, pp. 16-18.

[22] K.D. Young, V.I. Utkin, U. Ozguner, A control engineer's guide to sliding mode control, IEEE Transactions on Control Systems Technology 7 (3) (1999) $328-342$.

[23] G. Bartolini, L. Fridman, A. Pisano, E. Usai (Eds.), Modern Sliding Mode Control Theory. New Perspectives and Applications, in: Springer Lecture Notes in Control and Information Sciences, vol. 375, 2008.

[24] X.G. Yan, C. Edwards, Nonlinear robust fault reconstruction and estimation using a sliding mode observer, Automatica 43 (2007) 1605-1614

[25] C. Edwards, S.K. Spurgeon, R.J. Patton, Sliding mode observer for fault detection and isolation, Automatica 36 (4) (2000) 541-553.

[26] X.G. Yan, C. Edwards, Nonlinear robust fault reconstruction and estimation using a sliding mode observer, Automatica 43 (2007) 1605-1614.

[27] J.F. Bejarano, L. Fridman, A. Poznyak, Exact state estimation for linear systems with unknown inputsbased on hierarchical super-twisting algorithm, International Journal on Robust and Nonlinear Control 17 (18) (2007) 1734-1753.

[28] J.F. Bejarano, L. Fridman, A. Poznyak, Unknown input and state estimation for unobservable systems, SIAM Journal of Optimal Control 48 (2) (2009) $1155-1178$.

[29] A. Isidori, Nonlinear Control Systems, third ed., Springer Verlag, London, 1995.

[30] G. Bartolini, A. Pisano, E. Usai, Global stabilization for nonlinear uncertain systems with unmodeled actuator dynamics, IEEE Transactions on Automatic Control 46 (11) (2001) 1826-1832.

[31] X.G. Yan, C. Edwards, Nonlinear robust fault reconstruction and estimation using a sliding mode observer, Automatica 43 (2007) 1605-1614.

[32] G. Bartolini, A. Levant, A. Pisano, E. Usai, Higher-order sliding modes for output-feedback control of nonlinear uncertain systems, in: X. Yu, J. Xu (Eds.), Variable Structure Systems: Towards the 21-st Century, in: Lecture Notes in Control and Information Sciences, vol. 274, Springer Verlag, 2002, pp. 83-108.

[33] A. Levant, Sliding order and sliding accuracy in sliding mode control, International Journal of Control 58 (6) (1993) 1247-1263.

[34] G. Bartolini, A. Ferrara, E. Usai, Output tracking control of uncertain nonlinear second-order systems, Automatica 33 (12)(1997) $2203-2212$

[35] G. Bartolini, A. Ferrara, A. Pisano, E. Usai, On the convergence properties of a 2-sliding control algorithm for nonlinear uncertain systems, International Journal of Control 74 (2001) 718-731.

[36] G. Bartolini, A. Pisano, E. Usai, An improved second-order sliding mode control scheme robust against the measurement noise, IEEE Transactions on Automatic Control 49 (10) (2004) 1731-1737.

[37] C. Join, J.-C. Ponsart, D. Sauter, D. Theilliol, Nonlinear filter design for fault diagnosis: application to the three-tank system, IEE Proceedings IEEE Transactions on Control System Technology 13 (3) (2005).

[38] http://www.inteco.com.pl/index.php. 